\title{
Prediabetes Is Associated with HNF-4a P2 Promoter Polymorphism rs1884613: A Case-Control Study in Han Chinese Population and an Updated Meta-Analysis
}

\author{
Changyi Wang, ${ }^{1}$ Sihan Chen, ${ }^{1}$ Tao Zhang, ${ }^{1}$ Zhongwei Chen, ${ }^{1}$ Shengyuan Liu, ${ }^{1}$ \\ Xiaolin Peng, ${ }^{1}$ Jianping Ma, ${ }^{1}$ Xiaohong Zhong, ${ }^{2}$ Yanqiong Yan, ${ }^{2}$ Linlin Tang, \\ Yifeng Mai, ${ }^{3}$ Liyuan Han, ${ }^{4}$ and Shiwei Duan ${ }^{4}$ \\ ${ }^{1}$ Shenzhen Nanshan Center for Chronic Disease Control, Shenzhen 518054, China \\ ${ }^{2}$ Shenzhen Shekou People's Hospital, Shenzhen 518067, China \\ ${ }^{3}$ The Affiliated Hospital, School of Medicine, Ningbo University, Ningbo, Zhejiang 315000, China \\ ${ }^{4}$ School of Medicine, Zhejiang Provincial Key Laboratory of Pathophysiology, Diabetes Center, Ningbo University, \\ Ningbo 315211, China
}

Correspondence should be addressed to Sihan Chen; 14900365@qq.com, Liyuan Han; hanliyuan@nbu.edu.cn and Shiwei Duan; duanshiwei@nbu.edu.cn

Received 5 July 2014; Accepted 11 September 2014; Published 15 October 2014

Academic Editor: Stamatios Theocharis

Copyright (C) 2014 Changyi Wang et al. This is an open access article distributed under the Creative Commons Attribution License, which permits unrestricted use, distribution, and reproduction in any medium, provided the original work is properly cited.

Background. Controversy remains for the association between hepatocyte nuclear factor $4 \alpha$ (HNF- $4 \alpha$ ) P2 promoter polymorphism rs1884613 and type 2 diabetes (T2D). There was no association test of this polymorphism with prediabetes and T2D in the Chinese population. Moreover, an updated meta-analysis in various ethnic groups is needed to establish the contribution of rs 1884613 to T2D risk. Methods. Using the Sequenom MassARRAY platform approach, we genotyped rs 1884613 of $H N F-4 \alpha$ in the P2 promoter region among 490 T2D patients, 471 individuals with prediabetes, and 575 healthy controls. All the individuals were recruited from 16 community health service centers in Nanshan district in Shenzhen province. Using STATA 11.0 software, meta-analysis was performed to summarize the overall contribution of rs1884613 to T2D risk. Results. Polymorphism rs1884613 was associated with genetic susceptibility to prediabetes in the whole samples $(\mathrm{OR}=1.40,95 \% \mathrm{CI}=1.16-1.68, P=0.0001)$ and the female subgrouped samples $(\mathrm{OR}=1.48,95 \% \mathrm{CI}=1.14-1.92, P=0.003)$ after adjusting for age and body mass index $(\mathrm{BMI})$. In contrast, there was no association of rs1884613 with T2D in the whole samples and male in our case-control study and meta-analysis. Conclusions. Our results suggest that rs1884613 contributes to susceptibility to prediabetes, whereas this polymorphism may not play an important role in the development of T2D.

\section{Introduction}

The prevalence rate of type 2 diabetes (T2D) is increasing rapidly, with $8.4 \%$ of the global prevalence rate of the adults according to the International Diabetes Federation [1]. T2D is a complex metabolic disorder with strong familial aggregation and genetic predisposition [2]. Although genetic studies have made substantial progress in exploring the roles of genes in the development of T2D, the current findings only explain a small proportion of heritability [3,4]. Meanwhile, controversies remain for the validation of candidate genes in diverse populations with different genetic backgrounds.
As a pivotal transcription factor in the progress and functions of the pancreatic $\beta$-cells, hepatocyte nuclear factor $4 \alpha(H N F-4 \alpha)$ is differentially expressed in many tissues including the liver and pancreas [5]. HNF- $4 \alpha$ spans $29 \mathrm{~kb}$ on chromosome 20q13.1-13.2 [6], which is a known T2D susceptibility locus. HNF- $4 \alpha$ was shown to play a significant role in the development of maturity-onset diabetes of the young-1, which is characterized by impaired insulin secretion [7].

Polymorphism rs1884613 is located in the HNF-4 $\alpha$ P2 promoter, which is about $45.6 \mathrm{~kb}$ upstream of the transcription start site and regulates the primary splicing form of $H N F-4 \alpha$ in the pancreatic $\beta$-cell $[8,9]$. Strong association 
was found between the haplotype of P2 promoter variants and T2D risk; however no significant associations for the haplotypes of other variants outside the P2 promoter region were found, suggesting that the $\mathrm{P} 2$ promoter region might be the susceptibility region for T2D [10].

P2 promoter polymorphism rs1884613 of $H N F-4 \alpha$ was tested with T2D susceptibility in various ethnicities but not in the Chinese Han population. Considering the diverse genetic background among different populations, for which they may have a different predisposing genetic risk, the influence of rs1884613 on T2D susceptibility in the Chinese population remained to be identified. Therefore we studied the association of rs1884613 among 490 T2D subjects, 471 prediabetes subjects, and 575 healthy controls to extend knowledge in different populations.

Winckler et al. demonstrated the significant association of rs1884613 in the P2 promoter region in >3,400 patients and controls from Sweden, Finland, and Canada; however they did not confirm the significant association in an additional sample of 4,470 patients from North America and Poland (trended in the opposite direction) [11]. The inconsistencies across studies may be due to the lack of power and inadequate sample size among individual studies; therefore it is necessary to conduct a comprehensive meta-analysis to confirm the relevant relationship. In addition to the present case-control study, we also conducted an updated meta-analysis in several ethnic groups with all published studies and our case-control data.

\section{Research Design and Methods}

The participants in the current study came from 16 community health service centers (CHSC) in Nanshan district under the supervision of Shenzhen Nanshan Center for Chronic Disease Control. We applied a two-stage sampling method and a simple random procedure according to the sequence of computer-generated random numbers. Among those 1516 subjects, 490 had T2D (242 men and 248 women, mean age: $62.76 \pm 11.14$ years, mean body mass index (BMI): $24.95 \pm$ $3.46 \mathrm{~kg} / \mathrm{m}^{2}$ ), 471 were prediabetes subjects ( 230 men and 241 women, mean age: $61.39 \pm 11.43$ years, mean BMI: $25.28 \pm$ $3.82 \mathrm{~kg} / \mathrm{m}^{2}$ ), and 575 were healthy controls (286 men and 289 women; mean age: $57.94 \pm 10.81$ years; mean BMI: $23.52 \pm$ $\left.3.17 \mathrm{~kg} / \mathrm{m}^{2}\right)$.

T2D and prediabetes were diagnosed according to the criteria of the American Diabetes Association guidelines in 2010 [12]. Informed written consent was obtained from all subjects before participation. The study was approved by the Ethical Committee of Shenzhen Nanshan Center for Chronic Disease Control.

\section{Genotyping}

The blood samples $(5 \mathrm{~mL})$ were collected after overnight fasting at morning without stasis in EDTA vacutainers. Polymorphism rs1884613 was genotyped using Sequenom's MassARRAY iPLEX system according to the manufacturers' instructions. Primers for the PCR and single base extension were designed using the Sequenom software. DNA sequences of primers were $5^{\prime}$-ACGTTGGATGACTCTGTCGTGGCTCCAGTA- $3^{\prime}$ and $5^{\prime}$-ACGTTGGATGAATTGGCTTGTGGACATCCG-3'. Thermocycling was carried out under the following conditions: $94^{\circ} \mathrm{C}$ for $15 \mathrm{~min}$ followed by 45 cycles at $94^{\circ} \mathrm{C}$ for $20 \mathrm{~s}, 56^{\circ} \mathrm{C}$ for $30 \mathrm{~s}$, and $72^{\circ} \mathrm{C}$ for $1 \mathrm{~min}$, with a final incubation at $72^{\circ} \mathrm{C}$ for $3 \mathrm{~min}$.

\section{Statistical Analysis}

The age and BMI of involved individuals were described as means \pm SD. The differences of age and BMI among T2D subjects, prediabetes subjects, and healthy controls were tested using one-way ANOVA. Deviation of HardyWeinberg equilibrium (HWE) was assessed by $\chi^{2}$ test. To prevent the influence from genetic assumptions on the inheritance models, additive, recessive, and dominant models were applied for the association tests. Binary logistic regression analysis was used to calculate the odds ratios (ORs) and 95\% confidence intervals (CIs) after being adjusted by age and BMI. Bonferroni's adjustment was applied to the significance thresholds, and a $P$ value of $<0.008$ was adopted as the significant threshold (Table 2). Power analysis was simulated with the Power and Sample Size Calculation software [13]. All statistical tests were performed by SPSS program version 17.0 (SPSS, Chicago, IL).

Eligible studies were identified by searching PubMed, Embase, Cochrane Library, and Science Citation Index Expanded databases for articles published in English until July 10, 2014. We applied the following search terms: "HNF$4 \alpha$ " or "polymorphism" or "variant" or "rs1884613" and in combination with "diabetes" or "T2D." The reference lists in the involved articles were also checked for additional relevant publications. Two investigators independently extracted the following data: the first author's name, year of publication, country, ethnicity, source of controls, number of cases and controls, the available genotype, and allele frequencies. Any disagreement was resolved by discussion. ORs with $95 \%$ CIs were estimated to assess the strength of association; the significance was determined using the $Z$ test $(P<0.05$ was considered statistically significant). Subgroup analyses were performed based on ethnicity (Caucasian or Asian) and source of control groups (population- or hospital-based studies). Heterogeneity was measured using Cochran's $Q$ test and $I^{2}$ test [14]. If the heterogeneity was not significant $(P>0.10)$, the fixed-effect model was used to evaluate the summary ORs and 95\% CIs. Otherwise, the randomeffect model was applied. The $I^{2}$ was used to estimate the heterogeneity quantitatively, with $I^{2}<25 \%, 25-75 \%$, and $>75 \%$ represented as low, moderate, and high degrees of inconsistency, respectively. Meta-analysis was conducted with the STATA software (version 11; Stata Corporation, College Station, Texas).

\section{Results}

The characteristics of the case-control subjects were shown in Table 1. There was statistical significance in age and BMI between prediabetes versus controls, type 2 diabetes versus 
TABLE 1: Characteristics of the subjects included in this study.

\begin{tabular}{|c|c|c|c|c|c|c|}
\hline Characteristic & Controls & Prediabetes & Type 2 diabetes & P1 & P2 & P3 \\
\hline Number of subjects & 575 & 471 & 490 & & & \\
\hline Female/male & $289 / 286$ & $241 / 230$ & $248 / 242$ & 0.81 & 0.91 & 0.95 \\
\hline Age & $57.94 \pm 10.81$ & $61.39 \pm 11.43$ & $62.76 \pm 11.14$ & 0.001 & 0.055 & 0.001 \\
\hline BMI & $23.52 \pm 3.17$ & $25.28 \pm 3.82$ & $24.95 \pm 3.46$ & 0.001 & 0.143 & 0.001 \\
\hline \multicolumn{7}{|l|}{ Female } \\
\hline Age & $58.50 \pm 10.00$ & $61.66 \pm 10.43$ & $63.99 \pm 10.28$ & 0.001 & 0.01 & 0.001 \\
\hline BMI & $23.18 \pm 3.09$ & $25.18 \pm 4.04$ & $24.69 \pm 3.58$ & 0.001 & 0.12 & 0.001 \\
\hline \multicolumn{7}{|l|}{ Male } \\
\hline Age & $57.38 \pm 11.56$ & $61.10 \pm 12.42$ & $61.51 \pm 11.85$ & 0.001 & 0.70 & 0.001 \\
\hline BMI & $23.87 \pm 3.22$ & $25.37 \pm 3.59$ & $25.20 \pm 3.32$ & 0.001 & 0.59 & 0.001 \\
\hline
\end{tabular}

Groups were compared using one-way ANOVA.

P1: Prediabetes versus controls; P2: type 2 diabetes versus Prediabetes; P3: type 2 diabetes versus controls.

TABLE 2: Comparison of genotypic and allelic distribution of rs1884613 in the (HNF)- $4 \alpha$ gene among subjects with type 2 diabetes, Prediabetes, and healthy controls.

\begin{tabular}{|c|c|c|c|c|c|c|c|c|c|c|c|c|}
\hline \multirow{2}{*}{$\begin{array}{l}\text { HNF4A } \\
\text { rs1884613 }\end{array}$} & \multirow{2}{*}{$\mathrm{CC}$} & \multirow{2}{*}{ CG } & \multirow{2}{*}{ GG } & \multirow{2}{*}{$\mathrm{C}$} & \multirow{2}{*}{ G } & \multicolumn{2}{|c|}{ Additive model } & \multicolumn{2}{|c|}{ Dominant model } & \multicolumn{2}{|c|}{ Recessive model } & \multirow{2}{*}{ HWE } \\
\hline & & & & & & OR $(95 \% \mathrm{CI})$ & $P$ & OR $(95 \% \mathrm{CI})$ & $P$ & OR $(95 \% \mathrm{CI})$ & $P$ & \\
\hline Controls & 171 & 288 & 94 & 630 & 476 & & & & & & & 0.14 \\
\hline Prediabetes & 128 & 224 & 117 & 480 & 458 & $1.40(1.16-1.68)$ & $0.0001^{\mathrm{a}}$ & $1.47(1.10-1.96)$ & $0.008^{\mathrm{a}}$ & $1.68(1.22-2.32)$ & $0.001^{\mathrm{a}}$ & \\
\hline Type 2 diabetes & 148 & 260 & 79 & 556 & 418 & $1.09(0.82-1.32)$ & 0.33 & $1.21(0.92-1.60)$ & 0.16 & $1.00(0.70-1.41)$ & 0.99 & \\
\hline Female & $\mathrm{CC}$ & CG & GG & $\mathrm{C}$ & G & & & & & & & \\
\hline Controls & 92 & 145 & 43 & 329 & 231 & & & & & & & 0.25 \\
\hline Prediabetes & 71 & 108 & 60 & 250 & 228 & $1.48(1.14-1.92)$ & $0.003^{\mathrm{a}}$ & $1.55(1.04-2.30)$ & 0.02 & $1.91(1.20-3.04)$ & $0.006^{\mathrm{a}}$ & \\
\hline Type 2 diabetes & 79 & 136 & 30 & 294 & 196 & $1.08(0.54-1.43)$ & 0.54 & $1.31(0.89-1.94)$ & 0.16 & $0.81(0.47-1.38)$ & 0.44 & \\
\hline Male & $\mathrm{CC}$ & CG & GG & $\mathrm{C}$ & G & & & & & & & \\
\hline Controls & 79 & 143 & 51 & 301 & 245 & & & & & & & 0.33 \\
\hline Prediabetes & 57 & 116 & 57 & 230 & 230 & $1.33(1.02-1.73)$ & 0.035 & $1.41(0.93-2.15)$ & 0.09 & $1.50(0.96-2.34)$ & 0.07 & \\
\hline Type 2 diabetes & 69 & 124 & 49 & 262 & 222 & $1.11(0.85-1.44)$ & 0.42 & $1.14(0.77-1.71)$ & 0.49 & $1.15(0.73-1.82)$ & 0.54 & \\
\hline
\end{tabular}

${ }^{\mathrm{a}}$ Significant results.

All of the ORs (95\% CIs) and $P$ values were adjusted for age and BMI.

controls (Table 1). As shown in Table 2, genotype distribution of rs1884613 in each subgroup met HWE. The results of genotypic association analyses were shown in an additive model, dominant model, and recessive model, respectively. The $P$ values presented were corrected for multiple testing (with $P<0.008$ as significant threshold). According to power calculations, our sample size provided $>90 \%$ power (at $\partial=$ 0.05 ) to detect the relative associations for rs1884613 with prediabetes risk.

After adjusting for age and BMI, rs1884613 showed strong association with prediabetes in the whole samples (Table 2, $\mathrm{OR}=1.40,95 \% \mathrm{CI}=1.16-1.68, P=0.0001)$ and in female samples (Table 2, OR $=1.48,95 \% \mathrm{CI}=1.14-1.92, P=0.003$ ). However, no association was found for the relationship between rs1884613 and T2D in the whole and male samples under the Bonferroni's correction (Table 2).

As reported in Table 3, the minor allele frequencies of rs1884613 were different among various ethnic samples. The allele frequencies of rs1884613-G were 0.16 and 0.18 in the Scandinavia and Norwegian populations, in contrast to 0.46 and 0.43 in the Japanese and Han Chinese populations. Inconsistent results were reported for the association of rs1884613 with T2D; therefore it is necessary to conduct a meta-analysis combined with several ethnic groups together at the same time. As shown in Table 4, the current metaanalysis involved a total of 8 studies with 8569 T2D subjects and 8528 controls. Among the 8 studies, 5 were populationbased studies (including our study) [11, 15-17] and 3 were hospital-based studies $[10,18,19]$. There were 5 studies from Caucasian populations [11, 15-18] and 3 studies from Asian populations (including the present study) $[10,19]$ (Table 4).

No significant association was observed in the whole meta-analysis (Table 5 and Figure $1, \mathrm{OR}=1.05,95 \% \mathrm{CI}=$ $0.99-1.11, P=0.06, P_{\text {heterogeneity }}=0.03$, and $\left.I^{2}=54.7 \%\right)$. No significant associations were observed in the stratified analyses by source of controls and ethnicity (Table 5). 
TABLE 3: Comparison of minor allele frequency among studies.

\begin{tabular}{|c|c|c|c|c|c|}
\hline \multirow{2}{*}{ Rs1884613 } & \multicolumn{2}{|c|}{ Minor allele frequency } & \multirow{2}{*}{$P$ value } & \multirow{2}{*}{ OR (95\% CI) } & \multirow{2}{*}{ Ethnicity } \\
\hline & Case & Control & & & \\
\hline American Diabetes Association [12] & 0.21 & 0.16 & 0.01 & $1.34(1.07-1.66)$ & Scandinavia \\
\hline Dupont and Plummer [13] & 0.27 & 0.21 & 0.017 & $1.38(1.06-1.80)$ & Ashkenazi Jewish \\
\hline Silander et al. [15] & 0.171 & 0.172 & 0.98 & $0.99(0.73-1.33)$ & Caucasian \\
\hline \multirow{6}{*}{ Boj et al. [8] } & 0.19 & 0.18 & 0.89 & $1.09(0.75-1.59)$ & Discordant sibs \\
\hline & 0.12 & 0.17 & 0.25 & $0.67(0.41-1.12)$ & Canada \\
\hline & 0.20 & 0.19 & 0.73 & $1.11(0.89-1.39)$ & Scandinavia \\
\hline & 0.19 & 0.15 & 0.12 & $1.25(0.99-1.58)$ & Sweden \\
\hline & 0.15 & 0.17 & 0.07 & $0.85(0.73-0.99)$ & GCI USA \\
\hline & 0.17 & 0.18 & 0.66 & $0.93(0.79-1.09)$ & GCI Poland \\
\hline Winter [7] & 0.482 & 0.469 & 0.71 & $1.05(0.79-1.39)$ & Japanese \\
\hline Love-Gregory et al. [16] & 0.474 & 0.477 & 0.89 & $1.01(0.88-1.15)$ & Japanese \\
\hline Higgins and Thompson [14] & 0.198 & 0.181 & 0.02 & $1.17(1.03-1.35)$ & Norwegian \\
\hline The present study & 0.43 & 0.43 & 0.33 & $1.09(0.82-1.32)$ & Chinese \\
\hline
\end{tabular}

GCI: Genomics Collaborative, Inc.

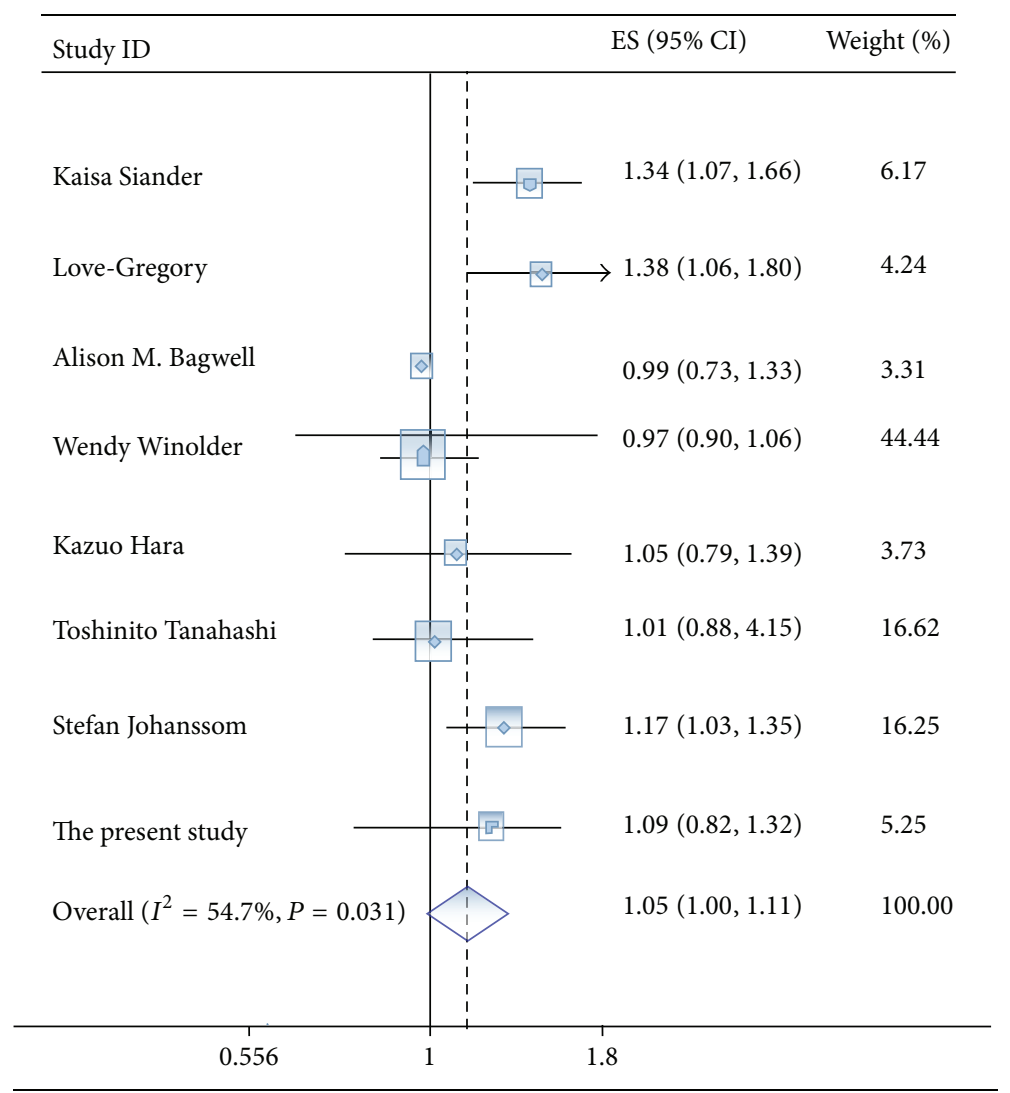

FIGURE 1: Meta-analysis of rs1884613 and type 2 diabetes risk under an additive model.

Significant heterogeneity was found in the whole metaanalysis and the subgroup meta-analysis (White and population-based studies). The Begg's and Egger's tests were performed to assess the publication bias, and no evidence of publication bias was observed (Table 5).

\section{Discussion}

We performed a case-control study to identify the effect of rs1884613 of $H N F-4 \alpha$ gene on prediabetes and T2D susceptibility in Han Chinese population. Our results suggest 


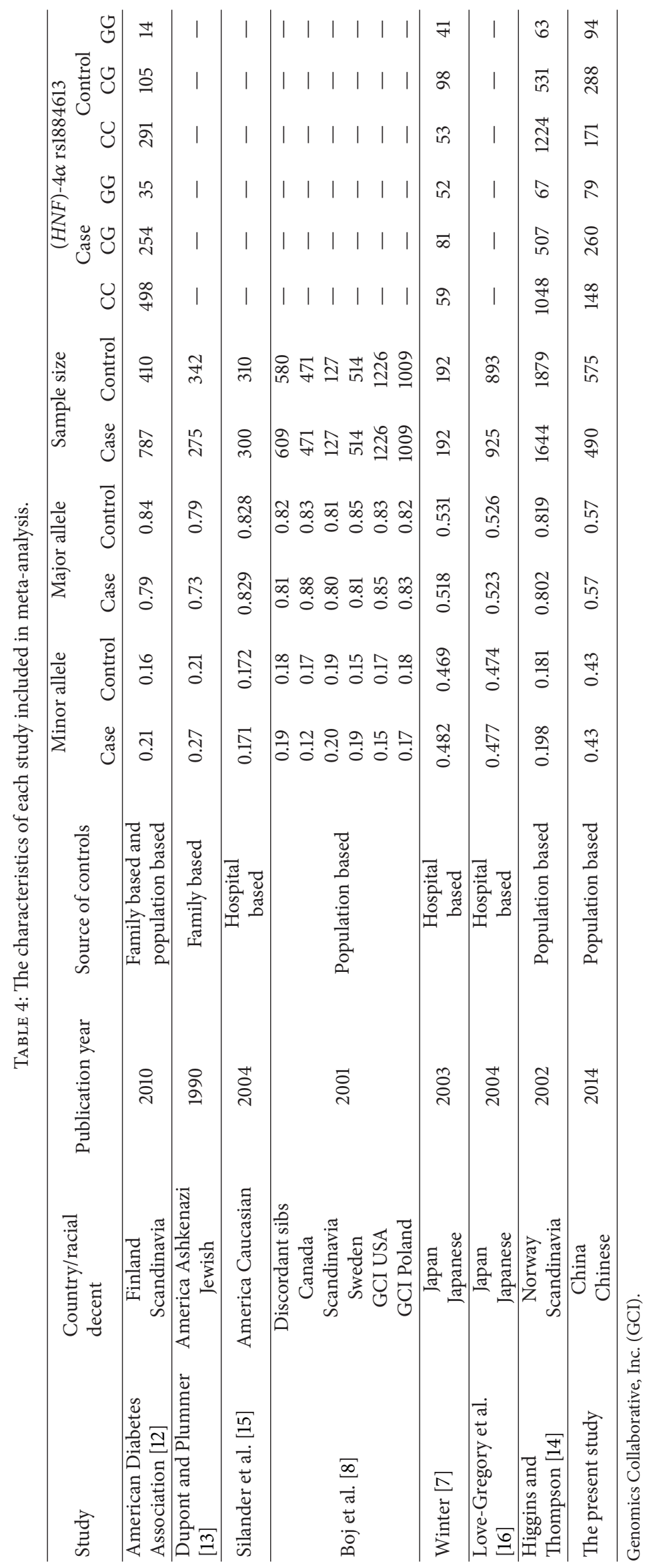


TABLE 5: Meta-analysis of association between rs1884613 and type 2 diabetes risk under an additive model.

\begin{tabular}{lcccccccc}
\hline & Number of studies & \multicolumn{2}{c}{ Test of association } & \multicolumn{2}{c}{ Test of heterogeneity } & \multicolumn{2}{c}{ Test of publication bias } \\
& (Cases/controls) & OR $(95 \% \mathrm{CI})$ & $Z$ & $P$ & $P_{\text {heterogeneity }}$ & $I^{2}(\%)$ & Begg's & Egger's \\
\hline All & $8(8569 / 8528)$ & $1.05(0.99-1.11)$ & 1.92 & 0.06 & 0.03 & $54.7 \%$ & 0.71 & 0.11 \\
Asian & $3(1607 / 1660)$ & $1.03(0.92-1.14)$ & 0.57 & 0.57 & 0.85 & $0 \%$ & 0.60 & 0.36 \\
White & $5(6962 / 6868)$ & $1.06(0.99-1.12)$ & 1.81 & 0.07 & 0.005 & $73.3 \%$ & 0.80 & 0.17 \\
Hospital based & $3(1417 / 1395)$ & $1.01(0.90-1.13)$ & 0.23 & 0.81 & 0.95 & $0 \%$ & 0.60 & 0.84 \\
Population based & $5(7152 / 7133)$ & $1.15(0.99-1.33)$ & 1.94 & 0.05 & 0.005 & $72.9 \%$ & 0.46 & 0.06 \\
\hline
\end{tabular}

that rs1884613 contributes to prediabetes susceptibility in the whole and female samples. Both our case-control association and the follow-up meta-analysis are unable to support an important role of rs1884613 in the risk of T2D. Our casecontrol study has $>90 \%$ power to detect an association with an OR of 1.40 or greater. To the best of our knowledge, this is the first report for the association of $H N F-4 \alpha$ rs1884613 with prediabetes and T2D in Han Chinese population.

It should be noted that Damcott et al. also conducted a study to explore the SNPs across the promoter and coding regions of $H N F-4 \alpha$ with T2D in 137 T2D, 139 prediabetes, and 342 healthy individuals; they only found that rs1884614 was significantly associated with T2D and the combined samples of T2D and prediabetes [20]. However they did not analyze the data according to gender; therefore we cannot compare the results concerning gender between our study and their study.

Consistent with our study, Winckler et al. and Bagwell et al. reported no association of $H N F-4 \alpha$ polymorphisms (including rs1884613) with T2D in Scandinavians and Caucasian Americans [11, 18], although several other studies found the significant association with risk of T2D in the Finnish population [16], the Ashkenazi Jews [15], the Mexican Americans [21], and the Norwegians [17]. The opposite findings among different studies may be due to different sample size, environmental factors, and different genetic backgrounds. In the studies by Silander et al. and LoveGregory et al., the cases were mainly from families with history of $\mathrm{T} 2 \mathrm{D}$, which may overestimate the contribution of rs 1884613 to T2D $[15,16]$. It is noteworthy that the minor allele frequencies among studies are significantly different. The $\mathrm{G}$ allele for rs 1884613 had a frequency of 0.16 in diabetic Scandinavia [15], 0.18 in diabetic Norwegian [17], 0.27 in diabetic Ashkenazi Jewish [16], 0.48 in diabetic Japanese [10], and 0.43 in diabetic Chinese of the present study. The genetic predisposition to T2D in different populations may be related to the differences in allele frequencies.

There may be some unknown susceptibility functional variants that are in linkage disequilibrium (LD) with rs1884613 in the populations with positive findings. The conflicting results in other studies may be due to the varying $\mathrm{LD}$ patterns in different tested populations. Polymorphisms rs4810424, rs1884613, rs1884614, and rs2144908 were in almost complete LD in the Finnish and Ashkenazi Jewish populations; however the four polymorphisms are not situated in any confirmed functional regions [15], suggesting that these four polymorphisms may be in strong LD with a yet unknown but functional polymorphism [22]. The discrepancy among studies may also be attributable to the false-positive or false-negative results.

$H N F-4 \alpha$ T130I polymorphism is a rare nonsynonymous variant that was shown to regulate $H N F-4 \alpha$ gene expression and thus was shown to be associated with T2D risk [23, 24]. Furthermore, the interaction between PPARG Pro12Ala and $H N F-4 \alpha$ rs 2144908 was shown to postchallenge insulin secretion [25]. Trip3 gene was not shown to be a susceptibility gene of early-onset T2D in Japanese population but might play an important role in glucose metabolism through regulating the transcription activity of $H N F-4 \alpha$ [26]. The above observations imply that the contribution of $H N F-4 \alpha$ variants to $\mathrm{T} 2 \mathrm{D}$ risk is not alone.

In order to confirm the association between rs1884613 and T2D susceptibility, we performed a meta-analysis among 8569 cases and 8528 controls from 8 studies including ours. Our meta-analysis showed there was no significant association between rs1884613 and T2D risk. Compared with the previous two meta-analyses $[11,17]$, the present one was involved in more studies. The meta-analysis by Johansson et al. only integrated results from homogenous populations of Scandinavians, although they detected a positive association but with a relatively small effect size (4000 cases and 7571 controls, $\mathrm{OR}=1.14, P=0.0004)$ [17]. The meta-analysis by Winckler et al. was involved with 7883 people and failed to confirm the significant association between rs1884613 and $\mathrm{T} 2 \mathrm{D}$ risk $(\mathrm{OR}=0.59,95 \% \mathrm{CI}=0.90-1.06)[11]$.

There are some limitations to be noted. Firstly, we only genotyped rs1884613 under the hypothesis that rs1884613 may be in $\mathrm{LD}$ with other $\mathrm{P} 2$ promoter variants. It may not stand for the whole P2 promoter variants in the Han Chinese population, although previous findings have shown the P2 promoter variants are in high LD with each other in Europeans [16]; therefore the SNPs across the promoter and coding regions of $H N F-4 \alpha$ with T2D and prediabetes should be further evaluated in future studies with different populations. Secondly, some populations in our metaanalysis consisted of the T2D cases from families with history of T2D. This may have confounded the results of the current meta-analysis. Thirdly, due to a lack of the detailed genotype frequency in most studies, the current meta-analysis only adopted the additive model that may not be the best heritable mode for this polymorphism. Fourthly, only a few studies had prediabetes subjects; therefore we were not able to perform the meta-analysis between rs1884613 and prediabetes risk. Last but not least, large heterogeneity was 
observed in the population-based subgroup meta-analysis. Since the hospital-based studies usually tend to have higher heterogeneity, the high heterogeneity in population-based studies may be due to the fact that some of the patients came from the families with history of T2D.

In conclusion, our results suggest that rs1884613 of HNF$4 \alpha$ is associated with prediabetes in Han Chinese population, whereas this polymorphism may not play an important role in the risk of T2D according to our case-control study and the updated meta-analysis. Future study is warranted to explore the $\mathrm{LD}$ patterns in the $\mathrm{P} 2$ region among the T2D patients from different ethnic populations.

\section{Conflict of Interests}

The authors declare that there is no conflict of interests or financial interests associated with this paper.

\section{Authors' Contribution}

Changyi Wang and Linlin Tang contributed equally to this work.

\section{Acknowledgments}

The authors are grateful to the participants, the doctors, and the nurses in the community health centers for their involvement in the data and sample collection. This research was supported by Grants from the National Natural Science Foundation of China (31100919, 81371469, and 81402745), Natural Science Foundation of Zhejiang Province (LR13H020003 and LQ13H260002), Zhejiang Province Scientific Research Projects of Education (no. Y201326971), K. C. Wong Magna Fund in Ningbo University and Ningbo Social Development Research Projects (2012C50032), Natural Science Foundation of Ningbo City (2014A610268), Ministry of Education, Humanities and Social Sciences Project (14YJC630046), Ningbo University Scientific Research Fund (XK11349), and Ningbo University Talent Project (ZX2012000046).

\section{References}

[1] G. Danaei, M. M. Finucane, Y. Lu et al., "National, regional, and global trends in fasting plasma glucose and diabetes prevalence since 1980: systematic analysis of health examination surveys and epidemiological studies with 370 country-years and 2.7 million participants," The Lancet, vol. 378, no. 9785, pp. 31-40, 2011.

[2] C. Dong, L. Tang, Z. Liu et al., "Landscape of the relationship between type 2 diabetes and coronary heart disease through an integrated gene network analysis," Gene, vol. 539, no. 1, pp. 3036, 2014.

[3] A. Brunetti, E. Chiefari, and D. Foti, "Recent advances in the molecular genetics of type 2 diabetes mellitus," World Journal of Diabetes, vol. 5, pp. 128-140, 2014.

[4] L. L. Tang, Q. Liu, S. Z. Bu et al., "The effect of environmental factors and DNA methylation on type 2 diabetes mellitus," Yi chuan $=$ Hereditas/Zhongguo yi chuan xue hui bian ji, vol. 35, pp. 1143-1152, 2013.
[5] L. Miquerol, S. Lopez, N. Cartier, M. Tulliez, M. Raymondjean, and A. Kahn, "Expression of the L-type pyruvate kinase gene and the hepatocyte nuclear factor 4 transcription factor in exocrine and endocrine pancreas," The Journal of Biological Chemistry, vol. 269, no. 12, pp. 8944-8951, 1994.

[6] A. Argyrokastritis, S. Kamakari, M. Kapsetaki, A. Kritis, I. Talianidis, and N. K. Moschonas, "Human hepatocyte nuclear factor-4 (hHNF-4) gene maps to 20q12-q13.1 between PLCG1 and D20S17," Human Genetics, vol. 99, no. 2, pp. 233-236, 1997.

[7] W. E. Winter, "Newly defined genetic diabetes syndromes: maturity onset diabetes of the young," Reviews in Endocrine and Metabolic Disorders, vol. 4, no. 1, pp. 43-51, 2003.

[8] S. F. Boj, M. Parrizas, M. A. Maestro, and J. Ferrer, "A transcription factor regulatory circuit in differentiated pancreatic cells," Proceedings of the National Academy of Sciences of the United States of America, vol. 98, no. 25, pp. 14481-14486, 2001.

[9] H. Thomas, K. Jaschkowitz, M. Bulman et al., "A distant upstream promoter of the HNF- $4 \alpha$ gene connects the transcription factors involved in maturity-onset diabetes of the young," Human Molecular Genetics, vol. 10, no. 19, pp. 2089-2097, 2001.

[10] K. Hara, M. Horikoshi, H. Kitazato et al., "Hepatocyte nuclear factor- $4 \alpha$ P2 promoter haplotypes are associated with type 2 diabetes in the Japanese population," Diabetes, vol. 55, no. 5, pp. 1260-1264, 2006.

[11] W. Winckler, R. R. Graham, P. I. W. de Bakker et al., "Association testing of variants in the hepatocyte nuclear factor $4 \alpha$ gene with risk of type 2 diabetes in 7,888 people," Diabetes, vol. 54 , no. 3 , pp. 886-892, 2005.

[12] American Diabetes Association, "Diagnosis and classification of diabetes mellitus," Diabetes care, vol. 33, supplement 1, pp. S62-S69, 2010

[13] W. D. Dupont and W. D. Plummer Jr., "Power and sample size calculations. A review and computer program," Controlled Clinical Trials, vol. 11, no. 2, pp. 116-128, 1990.

[14] J. P. Higgins and S. G. Thompson, "Quantifying heterogeneity in a meta-analysis," Statistics in Medicine, vol. 21, no. 11, pp. 1539$1558,2002$.

[15] K. Silander, K. L. Mohlke, L. J. Scott et al., "Genetic variation near the hepatocyte nuclear factor-4 alpha gene predicts susceptibility to type 2 diabetes," Diabetes, vol. 53, no. 4, pp. 1141-1149, 2004.

[16] L. D. Love-Gregory, J. Wasson, J. Ma et al., "A common polymorphism in the upstream promoter region of the hepatocyte nuclear factor- $4 \alpha$ gene on chromosome $20 \mathrm{q}$ is associated with type 2 diabetes and appears to contribute to the evidence for linkage in an ashkenazi jewish population," Diabetes, vol. 53, no. 4, pp. 1134-1140, 2004.

[17] S. Johansson, H. Ræder, S. A. Eide et al., "Studies in 3,523 Norwegians and meta-analysis in 11,571 subjects indicate that variants in the hepatocyte nuclear factor $4 \alpha$ (HNF4A) P2 region are associated with type 2 diabetes in scandinavians," Diabetes, vol. 56, no. 12, pp. 3112-3117, 2007.

[18] A. M. Bagwell, J. L. Bento, J. C. Hychaleckyj, B. I. Freedman, C. D. Langefeld, and D. W. Bowden, "Genetic analysis of HNF4A polymorphisms in Caucasian-American type 2 diabetes," Diabetes, vol. 54, no. 4, pp. 1185-1190, 2005.

[19] T. Tanahashi, D. Osabe, K. Nomura et al., "Association study on chromosome 20q11.21-13.13 locus and its contribution to type 2 diabetes susceptibility in Japanese," Human Genetics, vol. 120, no. 4, pp. 527-542, 2006. 
[20] C. M. Damcott, N. Hoppman, S. H. Ott et al., "Polymorphisms in both promoters of hepatocyte nuclear factor $4-\alpha$ are associated with type 2 diabetes in the Amish," Diabetes, vol. 53, no. 12, pp. 3337-3341, 2004.

[21] D. M. Lehman, D. K. Richardson, C. P. Jenkinson et al., "P2 promoter variants of the hepatocyte nuclear factor $4 \alpha$ gene are associated with type 2 diabetes in Mexican Americans," Diabetes, vol. 56, no. 2, pp. 513-517, 2007.

[22] S. K. Hansen, C. S. Rose, C. Glümer et al., "Variation near the hepatocyte nuclear factor (HNF)- $4 \alpha$ gene associates with type 2 diabetes in the Danish population," Diabetologia, vol. 48, no. 3, pp. 452-458, 2005.

[23] B. Jafar-Mohammadi, C. J. Groves, A. P. Gjesing et al., "A role for coding functional variants in HNF4A in type 2 diabetes susceptibility," Diabetologia, vol. 54, no. 1, pp. 111-119, 2011.

[24] J. Ek, C. S. Rose, D. P. Jensen et al., “The functional Thr130Ile and Val255Met polymorphisms of the hepatocyte nuclear factor- $4 \alpha$ (HNF4A): gene associations with type 2 diabetes or altered $\beta$ cell function among danes," The Journal of Clinical Endocrinology and Metabolism, vol. 90, no. 5, pp. 3054-3059, 2005.

[25] F. Wang, X.-Y. Han, Q. Ren et al., "Effect of genetic variants in KCNJ11, ABCC8, PPARG and HNF4A loci on the susceptibility of type 2 diabetes in Chinese Han population," Chinese Medical Journal, vol. 122, no. 20, pp. 2477-2482, 2009.

[26] H. Iwahashi, K. Yamagata, I. Yoshiuchi et al., "Thyroid hormone receptor interacting protein 3 (Trip3) is a novel coactivator of hepatocyte nuclear factor- $4 \alpha$," Diabetes, vol. 51 , no. 4 , pp. $910-$ 914, 2002. 


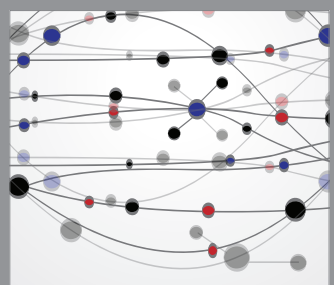

The Scientific World Journal
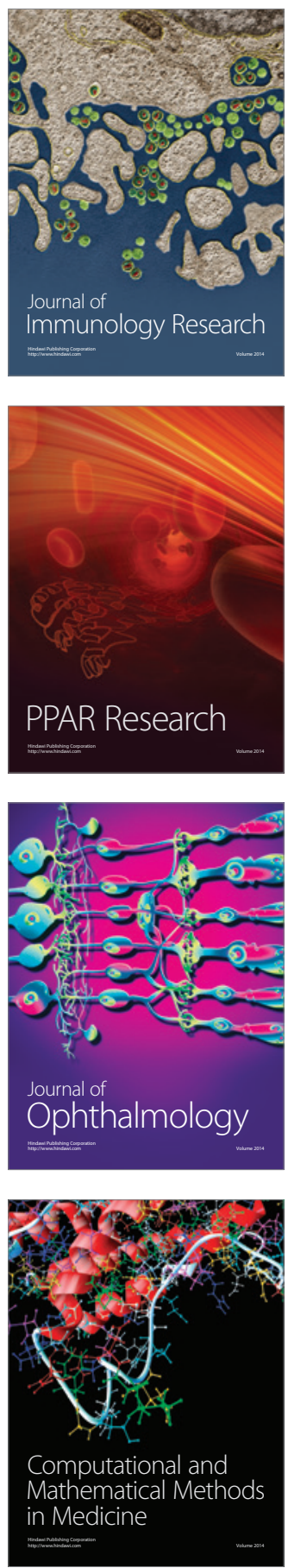

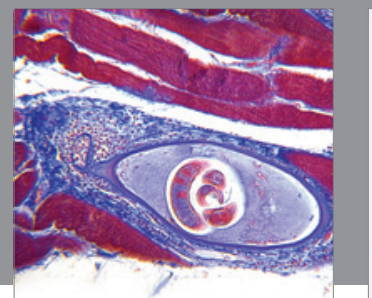

Gastroenterology

Research and Practice
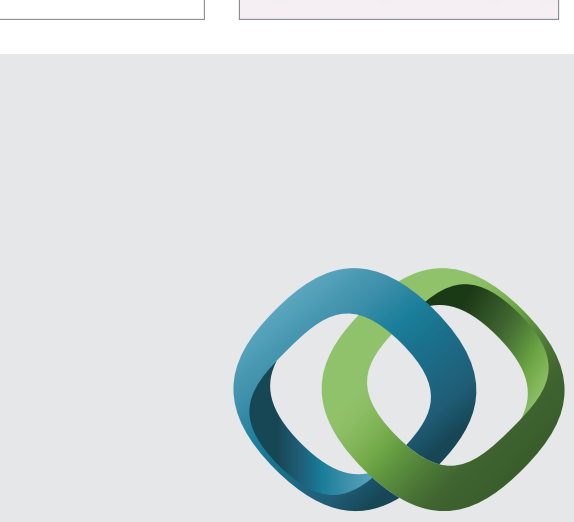

\section{Hindawi}

Submit your manuscripts at

http://www.hindawi.com
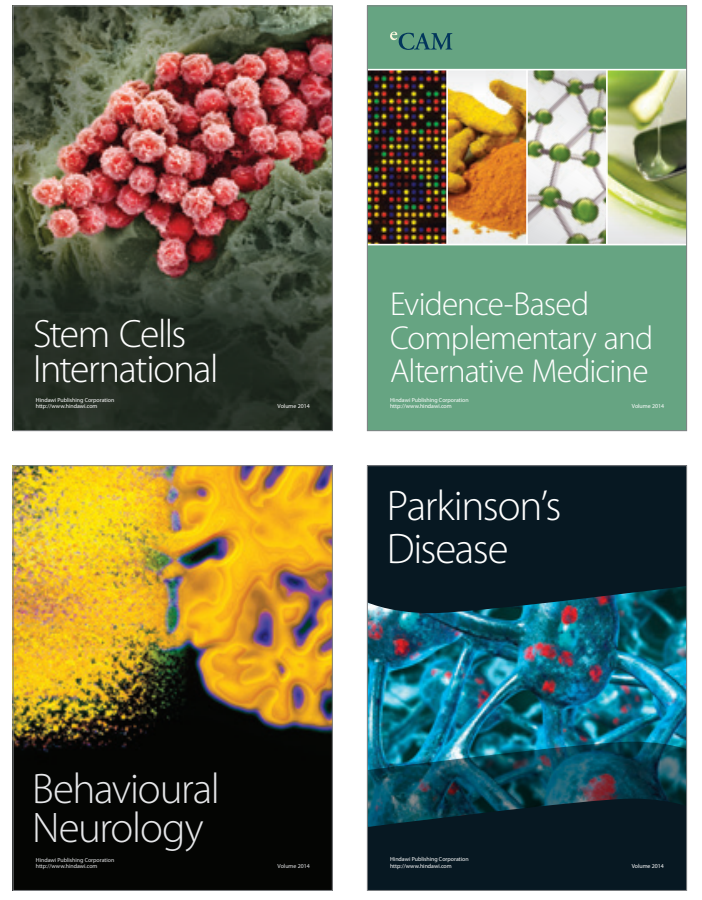
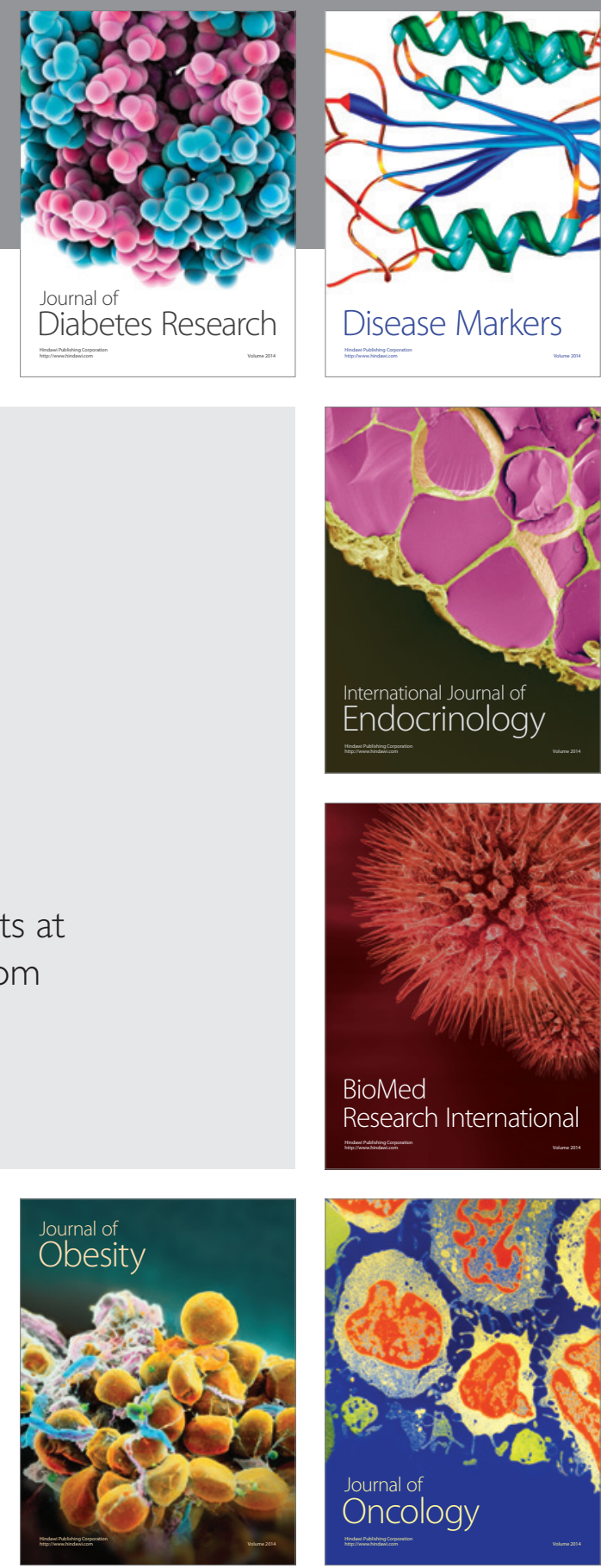

Disease Markers
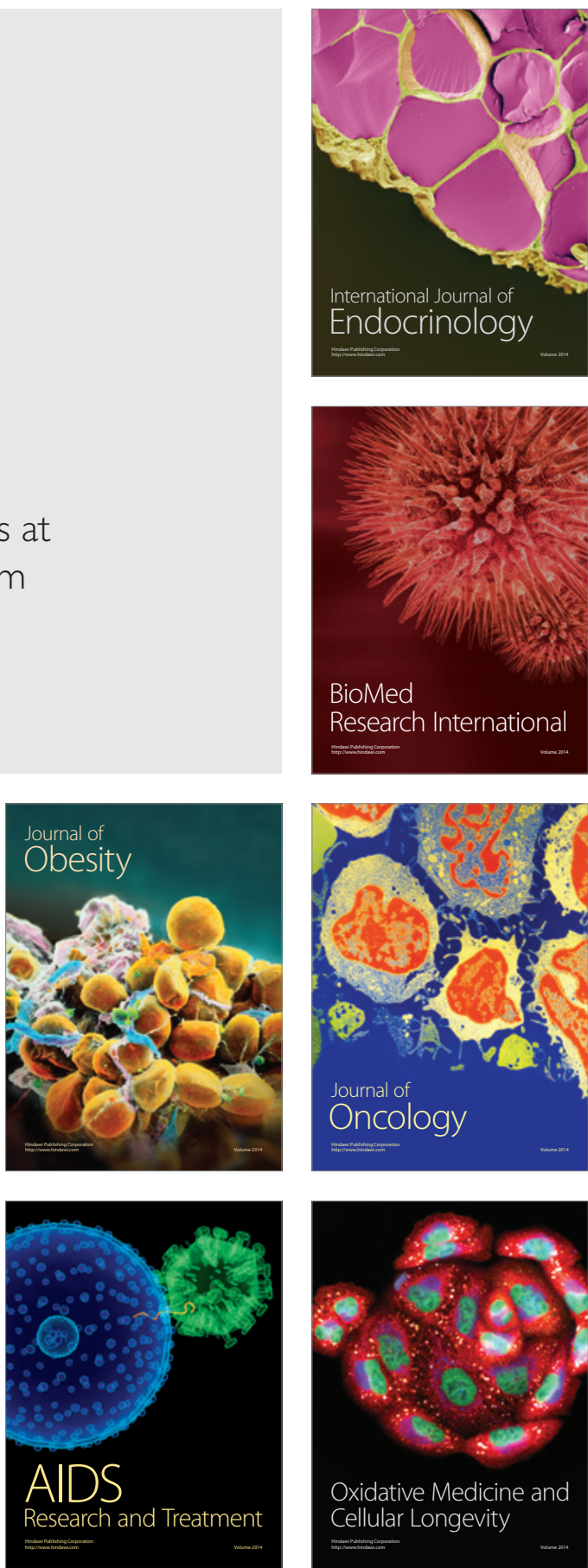